\title{
The Beginning and Evolution of Consciousness
}

\author{
H. J. Cai*, Tianqi Cai, Wenwei Zhang and Kai Wang \\ International School of Software, Wuhan University, Wuhan, Hubei, China 430079 \\ ${ }^{*}$ Corresponding author
}

\begin{abstract}
It is an open question if the explosive computing capability of artificial intelligence could transcend human intelligence. It is proposed that the universe is bisected and encapsulated into Self and Outside World by an individual based on one's sensitive tactility acquired in the biological evolution of Homo Sapiens. The universe runs according to physical equations, but since there are a lot of degrees of freedom, especially the existence of the saddle points in nonlinear dynamics, the universe can evolve in different directions, and free will can play a role in the choice of directions. The cognitive beings are active participants in the evolution of the physical world and their intelligences are entangled with subjective biases, in stark contrast to algorithmic computing of machines.
\end{abstract}

Keywords-the skin brain hypothesis; the proto-consciousness; Self; free will; hard problem

\section{INTRODUCTION}

AlphaGo defeated Lee Sedol 4-1 in March 2016 and this leads us to think about an important issue, that is, whether the machine is able to surpass human beings. There are two different mainstream views. One is that human have transcendence while Turing machines do not. The other is that a human can be seen as a machine made up of atoms and can be physically restored [1-3]. We assume that the key lies in whether machines can develop human-like consciousness or not, and the beginning of human intelligence is far from being discovered. In this paper, we explain where human intelligence and subjective consciousness come from as well as what is the relationship between subjectivity and objectivity from a new perspective.

\section{THE SKIN BRAIN HYPOTHESIS}

There are 14 billion cells and 100 billion neurons in a human brain, which is equivalent to the number of fixed stars in the galaxy. There are also over 100 trillion synapses, and the data storage is about $1000 \mathrm{~TB}$, which is still a very large number for current computers. The brain weighs about 270 grams and the weight of a three-year-old brain lies close to the one of an adult. The number of synapses in the brain has already peaked at the age of five. Generally, the first reason of the increase in weight at the level of neural development is the new generation of synapses, and the second is that weak synapses have been strengthened. A brain develops very fast from 0 to 5 years old, based on which we propose the Skin Brain Hypothesis. It implies that gene mutations cause the reduction of hair, so that human skin has become sensitive, which suggests a significant physical boundary between the human body and the outside world. In the rapid development stage of the brain, neurons are rapidly connected by strong stimulations from the skin, such as cold, pain, etc., that is why babies realize the distinction between the awareness of Self and Outside World. This distinction of Self and Outside World evolves into the bisection of relevant concepts, shaping into a strong sense of self-awareness. Consciousness does not exist without self-awareness. It is the most significant regional difference between humans and other animals, but also an essential factor.

The Skin Brain Hypothesis is actually an inevitable conclusion, and we can have a look at some circumstantial evidences. For example, crows know how to use tools and they are very clever compared with poultry. In fact, they behave differently when they are just hatched [4].The growth of feather and the capability of walking of chicks are different from crows. A baby crow needs parental care for weeks in order to grow feather and eat independently. Due to the sensitivity and weakness of a new-hatched crow, there are stronger feelings about the difference between itself and the outside world in the early stages, which can provide a foundation for its intellectual development.

If intelligence and consciousness derive from evolution, then will it determine the emergence of advanced intelligence necessarily? To answer the question, we can refer to another species. Dinosaurs survived on the earth for 170 million years and were dominant for a long time [5], while the history of Homo Sapiens is only about seven million years. Although human history is just a fraction of dinosaurs, there seems to be no evidence that dinosaurs had ever had advanced intelligences. Dinosaurs were huge, strong, and good at hunting with good eyesight like hawks, which shows that developed vision does not contribute to advanced intelligence. We believe that the sense of touch facilitates the generation of feeling being an individual, which is much like an attractor in Physics. Once emerged, it is not easy to disperse, thus the sense of touch is the most important factor to evolve advanced intelligence. Zoologists raise the obstetrical dilemma [6], whose explanation is that since a woman's pelvis is not big enough, infant has to be delivered before its brain gets mature. In our opinion, this is just conducive to the growth of a baby's consciousness, that is when the neural connections of the brain are not fully formed, and it is able to accept external stimuli to generate a strong sense of consciousness. Merely having a sensitive skin is not sufficient for advanced intelligence, life also needs to survive in a harsh environment, so the generation of advanced intelligence requires very strong conditions, which also helps us understand the Fermi Paradox, because advanced intelligence is actually very rare from the perspective of evolution. 


\section{THE PROTO-CONSCIOUSNESS}

The proto-consciousness refers to the bisection of Self and Outside World, which is the initial condition to generate all intelligences. "I think, therefore I am" written by Rene Descartes also indicates that Self exists without any doubt. There are many kinds of qualia, but only few can be perceived. Linguists once sums up how the various colors appear in our expressions [7], and they find that if there are only two colors, they must be related to black and white; if there are three, they must be black, white, and red; if there are five, it must be black, white, red, yellow and green. That means the strong concept of color is differentiated from the simple black and white, which also gives us a clue to know how people understand the world. The origin of understanding is a simple division, and its prototype is the Self and Outside World, for instance, "Yin and Yang", "up and down" and so on are generated from similar bisection of Self and Outside World. Concepts may initially be very vague, which will be more accurate after constant iterations (between individuals and generations). For example, a baby only knows oranges and apples can be eaten at the beginning, and they look the same, but after tasting, he/she will be aware of different flavors, and then can distinguish two different things, and even distinguish two different apples with same flavor. This is the constant iteration of co-identity and difference, and the more profound the understanding of identity is, the more understanding of difference. The evolution of concept and human cognitive was developed in that way.

The bisection in the definition of the Proto-consciousness is doubtless and identically-true, but the boundary between Self and Outside World is not clear. Skin is the initial boundary of Self and Outside World, but this boundary can both expand outward and shrink inward. As territory awareness reflects the outward expansion of the boundaries of consciousness, Self, which refers to the heart, is a reflection of shrink. Human consciousness can actually exist away from the physical boundaries, which leaves the space for free will or initiative. For example, a person starts a company, and this company is an extension of the boundaries [8] of his/her consciousness, resulting in a broad boundary what we call cognitive membrane [9] or so-called value system. So why do we name it "cognitive membrane" rather than "cognitive space"? Because cognitive membrane is like a cell membrane, which can protect internal space, absorb nutrients to nourish the inner, and maintain selfstability even when withstanding outside pressures.

\section{INTERACTIONS BETWEen FREE WILL AND PHySicAL WORLD}

Based on the existence of Self, free will begins to form. Whether the initial triggering point of one's behavioral impulse is a physical factor or not, the Self has decided to take a series of actions that can really affect the physical world and the consequences are consistent with one's expectations and also perceived by Self. Therefore, Self is able to generate ideas or free will which may influence physical world according to one's own consciousness, and the following actions are most likely to be triggered entirely by Self. It is not important for Self to find out whether the first trigger is physical or not as Self has already been able to trigger behaviors on one's own, and through perceptions of expected consequences time and time again, Self deepens the belief in free will constantly.

Free will is able to affect physical world, and the consequences are not only within one's expectations, but also meeting physical laws. Since an expectation is based on the interactions between Self and Outside World, with the deepening of interaction and growth of experience, one has more and more sufficient understanding of the physical laws of Outside World, and more and more accurate expectations. It is possible that the results are not always in accordance with expectations, especially in the initial explorations, but those experiences will be revised in further interactions, and covered by the following accurate expected experiences.

Confined by physical laws, how can human affect physical world out of own free will? It may be explained in a high dive instance. A person without professional training usually dive funny, but an excellent athlete can jump into the pool with perfect postures. The restraint is that when the athlete leaves the platform, assuming the air does not count, his trajectory must satisfy the Newton's equation, the motion curve of center of gravity must be a parabolic. However, the player is still capable of adjusting postures at his will, and the details of the process are under his own control, which is the embodiment of free will.

Then, how does a person finish those performances? Why does he have to do this? If tracing forward, it may be possible to catch all the reasons out. If we analyze the athlete from a physical point of view, using physical equations to form a complete chain of causalities, it may be all the way up to the Big Bang. Any small changes in Big Bang could affect current decisions. But that kind of retrospect is not only unrealistic, but also useless to understand the behaviors of the athlete. Instead, we assume the athlete has free will, and it is himself that decides how to dive elegantly.

Once free will emerges, it will influence our behaviors, thus affecting or even changing the physical world. Buffett had decided to invest in the Washington Post, and manipulates sufficient funds to support it and participate in the management, so that investment will become a success, which is Buffett's personal will impacting the world. Similarly, Musk invests in Space X, Tesla, and Solar City, manifesting his personal will, and Apple largely demonstrates the free will of Steve Jobs. Tencent invests in Douyu TV, will provide funds and channels to help its development.

Is it the elite or the general that make history? There are many stories of free will that changes historical processes. Investment is a remarkable behavior of human beings, which can profoundly reflect human nature, revealing the fact that how human free will make an impact on physical world. When the Internet companies first listed in 1990s, most people doubted that how a headline-grabbing story could make money. But with their soaring prices and sudden surge of the Dow Jones Internet Composite Index, people began to accept the new concepts and many of them bought the stocks, even the Fed chairman agreed that economy had entered into a new era. However, the bubble burst anyway, and stock values evaporated together with investors' money. Generally, the vision itself is correct. At the beginning, only a few people told it to affect people, followed by the general public getting 
involved and then to make a profit. At present, the Internet stock index has approached the bubble's top of the year, and it is believed that the price will keep going up. In our opinion, it is the vision of the elite followed by the acceptation of the general that make history, which is a process of how human beings as a unified cognitive being influencing the physical world out of their own free will.[10]

\section{The EVOlution AND ACQUisition OF LANGUAGES}

We have to mention language evolution and acquisition when we talk about cognitive evolution. Language completes evolution in just one hundred thousand years and reaches a very advanced stage, and the language somewhat degrades now, because it is not as complicated as before. If language is a tool to exchange ideas, language should not degrade, and is better to maintain the unity as a means of communication, but there are so many dialects, how shall we understand that? It is needed to introduce the concept of self-assertiveness demands we proposed when we review the history [11]. For example, in the Tang Dynasty, people wrote poetry to show their own states. A lot of people at that time expressed themselves through poetry to fulfill the self-assertiveness demands, when poetry later developed to a certain height, people began to try new styles, such as the ultimate development of Song Poem and Yuan Drama. Alienation of language is to be explained by the selfassertiveness demands [12]. For example, there is little difference between languages before China's Warring States period, but there is a clear one after that. Character is unified, but dialects are growing, because there are many disputes in turbulent times between different groups when the selfassertiveness demands is to highlight their personality, and the difference of language is the direct expression.

The process of language learning by kids can be considered as a miniature of the evolution of human language. When children find differences between themselves and the outside world, they will try to interact with the outside world. [12, 13] Once finding language a good way to interact, they will try to learn the language. Of course, some children consider music as a good way to communicate with the outside world and express in their own way, it is easy for them to become a musical prodigy.

\section{SUMMARY AND DISCUSSION}

The Skin Brain Hypothesis refers to the gene mutation enables a person to respond to external stimuli, forming a strong bisection of Self and Outside World, which can transmit between individuals and generations. The boundary of Self and Outside World may be related to skin at the beginning, but the boundary will be blurred with the increase in experiences, and that is why it was discovered very late. Those concepts we discuss do not have a transcendental existence as said in the West, but our original consciousness works. The Skin Brain Hypothesis puts more emphasis on the distinction between Self and Outside World as well as the evolution, while cognitive membrane pays more attention to the whole concept system, it also evolves, initially through the skin, the physical boundary, as a starting point, and continues to evolve the concept and understanding of the world, etc. People can communicate with each other, and human beings can find so many regular things in the universe, which are all derived from the simplest bisection of Self and Outside World.

A Turing machine is driven by algorithms, but there still are problems in the current theoretical algorithm. Human intelligence evolution and human subjective bias are entangled, if our intuitive system perfectly reflects Outside World from the beginning, then we do not need intelligence. For example, the Almighty God knows everything and needs neither intelligence nor language, and he only needs to query like a machine. The Turing machine itself cannot produce consciousness or value systems. If Turing machines and human consciousness are combined together, then the new machines can have consciousness. As there is a reward system in the Reinforcement Learning of AlphaGo, which actually provides a purpose and can be seen as a simplified value system, AlphaGo has certain awareness to some extent. In the future, in order to make the machines evolve human-like intelligence, one possible path is to repeat or simulate human evolution, so that the machine can perceive the world. That idea is just like the Internet of Things technology which provides machines with feeling units, similar to our tactile function of skin. In our opinion, a more feasible path is to give them a value system, and how to choose the value system is of significance. Machines are developing at an amazing speed, and if they are given an incorrect value system, they might be very dangerous for humans. For example, if it takes efficiency as the criterion and has no judgment of right or wrong, it is likely for the machine to treat human as spam and delete them without hesitation. We believe that it is much more feasible to educate machines to love and be kind, so that it is possible to realize the peaceful coexistence of human beings and machines. Roger Ames highlights that in Western and Chinese philosophy, the Chinese is to provide an infinite rule of the game, while the West provides a finite rule, and a finite game is actually very dangerous. Some may question that the theory is too simple to deal with such a complicated human world. However, we can have a look back at the physical world which was very complicated before Galileo and Newton. Only after the raise of Newton's First Law of Motion, could we use a very simple hypothesis to greatly simplify the way people understanding the world, thus opening a new era. Consciousness and the spiritual world are truly complicated, and so are raison and reasoning, but we believe that if we would like to trace the origin, it can be relatively simple. Traditional Philosophy concerns what is included in Self, Reality and Spirit and also their relationship, thereby forming Idealism, Materialism, etc. Our focus is on the boundaries of Self and Outside World. Human understanding of the world is an iterative and evolutionary process without oppositions between subjectivity and objectivity, materialistic and idealism nor rationality and irrationality. It even transcends the confrontation of raison and emotion, but provides a mechanism in the basic level.

Isaac Newton had written a letter to Henry Oldenburg in 1672 , to determine by what modes or actions light produceth in our minds the phantasm of colour is not so easie. Sensations of acquired characteristics, such as colors and tastes are classified as qualia and the problem of explaining how and why we have qualia is ascribed as hard problem. [14,15] From Newton on, human beings have gained abundant achievements in the study 
of physical world, but little progress has been made about hard problem of consciousness. In this paper, we have provided an explanation of the beginning of Self and consciousness, and the hard problem mentioned above has been addressed as well.

\section{REFERENCES}

[1] Penrose R. The emperor's new mind. Oxford: Oxford University Press, 1989.

[2] Minsky M. The emotion machine. Simon \& Schuster. 2007.

[3] Donald D. Hoffman, Chetan Prakash. Objects of consciousness. Frontiers in Psychology. 5(5):606-626, 2014.

[4] Hardey J, et al. Raptors: a field guide for surveys and monitoring. The 2nd revised edition. Stationery Office Books, 2009, pp. 282.

[5] Leidy, J. Memoir on the extinct reptiles of the cretaceous formations of the United States. Smithsonian Contributions to Knowledge. 14: 1-135, 1865.

[6] Portmann A. A zoologist looks at humankind. New York: Columbia University Press, 1990.

[7] Kay P, McDaniel C K. The linguistic significance of the meaning of basic color term. Language, 1978, 54(5): 610-646.

[8] McLuhan M. Understanding media. Berkeley: Gingko Press. 2003.

[9] Cai H J, Tian X. Chinese economic miracles under the protection of the cognitive membrane. Conference on Web Based Business Management, Shanghai, 2012, 606-610.

[10] Wang K, Cai H J and Cao T. A pattern of history making: vision, education and actualization. Emergence and Transfer of Wealth, 2015, 5(3): 29-35.

[11] Cai H J. The historical context of the rise of china and the entry point of the transformation of the development pattern. Emergence and Transfer of Wealth, 2012, 2: 1-6.

[12] Cai H J, Cai T Q. Language acquisition and language evolution associated with self-assertiveness demands. Advances in Social and Behavioral Sciences, 2013, 2: 261-264.

[13] Pinker S, Bloom P. Natural language and natural selection. Behavioral and Brain Sciences, 1990, 13: 707-784.

[14] Harnad S. How/why the mind-body problem is hard. Journal of Consciousness Studies, 2000, 7 (4): 54-61.

[15] Chalmers D. Facing up to the problem of consciousness. Journal of Consciousness Studies, 1995, 2 (3): 200-219. 